INPLASY

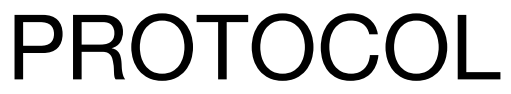

To cite: Fan et al. Influence of KIT mutations on prognosis of pediatric patients with corebinding factor acute myeloid leukemia: systematic review and meta-analysis. Inplasy protocol 202090019. doi: 10.37766/inplasy2020.9.0019

Received: 05 September 2020

Published: 05 September 2020

Corresponding author:

Fan Junjie

jjiefan@126.com

Author Affiliation:

Children's Hospital of

Soochow University

Support: None.

Review Stage at time of this submission: Piloting of the study selection process.

Conflicts of interest:

No conflicts of interest.

\section{Influence of KIT mutations on prognosis of pediatric patients with core-binding factor acute myeloid leukemia: systematic review and meta-analysis}

Fan, JJ1; Gao, L2; Chen, J3; Hu, SY4.

Review question / Objective: What is the role of KIT mutations on pediatric core-binding factor-acute myeloid leukemia (CBF-AML)?

Condition being studied: KIT signaling is involved in hematopoiesis. Mutations in the KIT gene are found in nearly a third of all core-binding factor-acute myeloid leukemia (CBF-AML) patients. The influence of KIT mutations on the prognosis of CBF-AML patients is currently unclear, with some studies reporting that these mutations are unfavorable and others reporting no obvious prognostic impact. It is necessary to identify the role of KIT mutations on the prognosis of pediatric CBF-AML.

INPLASY registration number: This protocol was registered with the International Platform of Registered Systematic Review and Meta-Analysis Protocols (INPLASY) on 05 September 2020 and was last updated on 05 September 2020 (registration number INPLASY202090019).

\section{INTRODUCTION}

Review question / Objective: What is the role of KIT mutations on pediatric corebinding factor-acute myeloid leukemia (CBF-AML)?
Condition being studied: KIT signaling is involved in hematopoiesis. Mutations in the KIT gene are found in nearly a third of all core-binding factor-acute myeloid leukemia (CBF-AML) patients. The influence of KIT mutations on the prognosis of CBF-AML patients is currently unclear, with some 
studies reporting that these mutations are unfavorable and others reporting no obvious prognostic impact. It is necessary to identify the role of KIT mutations on the prognosis of pediatric CBF-AML.

\section{METHODS}

Participant or population: Pediatric CBVAML patients with and without KIT mutations.

Intervention: We will investigating the effects of KIT mutations on the complete remission (CR), relapse, event-free survival (EFS), disease-free survival (DFS), and overall survival (OS) rates of pediatric CBFAML patients.

\section{Comparator: Patients with/without KIT mutations.}

Study designs to be included: Controlled clinical trials.

Eligibility criteria: The pediatric patients must be diagnosed as acute myeloid leukemia with CBF gene mutations characterized by the presence of one of the following two chromosomal rearrangements: $t(8 ; 21)(q 22 ; q 22)$ and inv(16) $(p 13 ; q 22) / t(16 ; 16)(p 13 ; q 22)$, which are usually shortened to $t(8 ; 21)$ and inv(16), respectively. We will analyse the role of KIT mutations on the prognosis of these patients by reviewing published controlled studies focus on this issue.

Information sources: We will do an electronic search of the PubMed, Cochrane Library, and Embase databases.

Main outcome(s): The complete remission (CR), relapse, event-free survival (EFS), disease-free survival (DFS), and overall survival (OS) rates of pediatric CBF-AML patients.

Quality assessment / Risk of bias analysis: To avoid bias, the eligibility of the studies will be determined by two co-authors (Junjie Fan and Li Gao), and any disagreements will be resolved by consultation with another author. The quality of the included studies will be assessed using the Newcastle-Ottawa scale (NOS). NOS scores will be independently calculated by two reviewers (Junjie Fan and Jing Chen). We will carry out "leave-one-out" sensitivity analysis: one study included in the meta-analysis be evaluated each time to confirm that our findings are not significantly changed by any single study.

Strategy of data synthesis: We will use the RevMan version $\mathbf{5 . 2}$ and Stata version 12.0 software packages. The CR, relapse, OS, EFS, and DFS rates will be calculated for each study. Risk ratios (RRs) will be used to assess the probabilities of $C R$ and relapse according to the presence or absence of KIT mutations. The OS, EFS, and DFS rates will be evaluated using hazard ratios (HRs). If Kaplan-Meier survival curves had been provided, the HRs will be calculated using the Parmar method, and the GetData Graph Digitizer software will be used to extract data from the survival curves. Initially, the fixed-effects model will be used to analyze all data, and then, the 12 statistic will be used to identify statistically significant heterogeneity between the studies. Heterogeneity will be quantified as mild, moderate, and severe, with upper limits of $25 \%, 50 \%$, and $75 \%$ for 12 , respectively. If the 12 value is $50 \%$ or lower, we will select the fixed-effects model; if not, we will choose the random-effects model.

Subgroup analysis: If there are necessary data available, we will do subgroup analyses with different comparators separately.

Sensibility analysis: Sensitivity analysis is used to evaluate the robustness of the main efficacy of indicators. We will carry out "leave-one-out" sensitivity analysis: one study included in the meta-analysis be evaluated each time to confirm that our findings are not significantly changed by any single study.

Language: English.

Country(ies) involved: China. 
Keywords: CBF-AML, pediatric, KIT, prognosis, meta-analysis.

Contributions of each author:

Author 1 - Junjie Fan - Conceptualization, Resources, Software, Drafting the manuscript.

Author 2 - Li Gao - Resources, Software, Drafting the manuscript.

Author 3 - Jing Chen - Resources, Software, Drafting the manuscript.

Author 4 - Shaoyan Hu - Conceptualization, Project administration. 